Background: Structural volume loss of cortical gray matter over time in schizophrenia has been widely reported (Vita et al. 2012), and may be more pronounced when the disorder has an onset prior to age 18 (Early Onset Psychosis, EOP; Arango et al. 2008). More recently, studies have focused on measures of cortical morphology. The single study in EOP so far has identified greater loss of cortical thickness $(\mathrm{CTH})$ in patients with schizophrenia over time (van Haren et al. 2011), whereas to our knowledge, no so far study has examined measures of surface area (SA) in EOP following a longitudinal design. We set out to examine measures of both CTH and SA in a sample of EOP at 10-year-follow-up.

Methods: Patients with EOP were recruited at first episode, matched by sex and age with healthy controls (HC) and re-assessed at 10 years. Subjects were evaluated clinically and structural $\mathrm{T} 1$ volumes were acquired using magnetic resonance imaging at baseline and 10-yearfollow-up. Images were preprocessed, segmented and analysed with FreeSurfer. Quality control procedure was carried out by two raters. Images were segmented and $\mathrm{CTH}$ and SA values were extracted for each parcellation employing Desikan-Killiany Atlas; these were grouped in frontal, occipital, temporal, parietal and cingulate lobes so as to reduce multiple comparisons. When group or group by time effects were detected, parcellations were individually examined. A linear mixed model was built using Stata IC 13.1 to evaluate the effect of group and time on $\mathrm{CTH}$ and SA, including hemisphere as fixed effects and correcting by total intracranial volume and setting a critical p-value of .05 .

Results: Thirty-nine subjects completed the follow-up. After removing 9 due to poor quality T1 images (technical problems, excess of movement), 28 subjects were finally included ( $13 \mathrm{EOP}, 15 \mathrm{HC})$. There were no significant differences in age ( $\mathrm{EOP}=26.9 \pm 0.6$ vs $\mathrm{HC}=27.2 \pm 0.3$ at follow-up) or sex distribution (\%female: $\mathrm{EOP}=43 \%$ vs $\mathrm{HC}=38 \%$ ) between groups. The distribution of diagnosis in the case group was: schizoaffective disorder $(n=5)$, bipolar disorder with psychotic features $(n=3)$, schizophrenia $(n=2)$ and others $(n=3)$.

There was a trend-level group effect in global CTH $(p=.07)$ which was significant in the frontal lobe $(\mathrm{p}=.014)$. EOP exhibited less CTH in the caudal middle frontal $(\mathrm{p}=.016)$ and pars opercularis $(\mathrm{p}=.03)$ and orbitalis $(\mathrm{p}=.007)$ of the inferior frontal gyrus. There was an effect of time in the parietal $(\mathrm{p}=.013)$ and occipital $(\mathrm{p}=.004)$ lobes consisting of thinner CTH at follow-up in both groups.

There were no differences in SA between groups. Both showed an increase in total SA $(p<.001)$ and for parietal $(p<.001)$, temporal $(p=.009)$ and occipital $(\mathrm{p}=.003)$ regions at follow-up. There a group by time effect in frontal SA, consisting of an increase over time in $\mathrm{HC}$ and a decrease in EOP $(p=.044)$, specifically in medial orbito-frontal cortex $(p=.039)$.

Discussion: Our results have identified: 1) thinner cortices in frontal regions in EOP compared to $\mathrm{HC}$, which seems to be constant over time; and 2) a decreased in SA in frontal areas in EOP along time, contrasting with $\mathrm{HC}$, whose frontal surface increased at follow-up. These findings are consistent with another study (Greenstein et al. 2006) which also reported reduced CTH in frontal areas in EOP during development, while we found no abnormalities in temporal regions (Vita et al. 2012). Despite the small sample size, to our knowledge this is the longest follow-up of an EOP sample employing magnetic resonance imaging so far.

\section{T172. MULTIMODAL QUANTIFICATION OF MEMORY CIRCUIT MICROSTRUCTURE IN FIRST EPISODE PSYCHOSIS}

Carolina Makowski*,1, Christine Tardif ${ }^{1}$, Gabriel Devenyi ${ }^{2}$, Robert Amaral ${ }^{3}$, Gabriella Buck ${ }^{3}$, Ridha Joober ${ }^{2}$, Ashok Malla ${ }^{2}$, Jai Shah ${ }^{2}$, Mallar Chakravarty², Martin Lepage ${ }^{2}$

${ }^{1}$ Mc Gill University; ${ }^{2}$ Mc Gill University, Douglas Mental Health University Institute; ${ }^{3}$ Douglas Mental Health University Institute
Background: Integrity of hippocampal subfield structure and associated limbic circuitry subserves various memory processes, a domain that is impaired in psychosis and an important predictor of functional outcome. We use a novel atlas that encapsulates both hippocampal subfields and surrounding white matter (WM), forming the 'memory circuit', to assess volumes with high-resolution MRI, and microstructure with quantitative T1 (qT1). Our aims were to examine 1) group by time interactions on memory measures and the memory circuit, and 2) explore the relationships between the chosen memory measures and limbic structures, informed by results from 1), in a longitudinal sample of first episode of psychosis (FEP) patients.

Methods: Nineteen FEP and 20 controls with baseline and 3-month follow-up data were included. Logical Memory and Visual Reproduction Subscales of the Weschler Memory Scale, and MRI scans on a 3T scanner were collected. High-resolution T2-weighted images $(0.64 \mathrm{~mm} 3)$ were input to the MAGeT Brain algorithm to obtain volumes of hippocampal subfields and surrounding WM, defined by fimbria, alveus, fornix, and mammillary bodies. Mean qT1 values for each hippocampal subfield and WM structure were sampled from MP2RAGE (1 mm3) qT1 maps. Linear mixed models were used to assess group by time interactions on memory measures, volumes and qT1. To begin, total hippocampal volumes and WM structure for each hemisphere were examined using a Bonferroni correction for multiple comparisons, followed by post-hoc tests of individual subfields and WM structures. Linear models were then used to assess relationships between baseline memory and change in anatomical measures of interest in FEP. Models controlled for sex, education, age, and brain volume.

Results: Significant group by time interactions emerged on bilateral mean WM qT1 (left: F1,65=9.3, p=.003; right: $F 1,65=10.6, p=.002$ ), where it was found that within the FEP group, qT1 (relaxation time in ms) increased over the 3-month follow-up period. Looking at WM structures separately, the interaction was driven by qT1 changes in fimbria, fornix, and mammillary bodies bilaterally ( $p$ 's $<.05$ ). No significant group by time interactions were found with respect to volumes or memory, although a trend-like group by time interaction on right fornix volume was found $(\mathrm{F} 1,64=5.6$, $\mathrm{p}$-uncorrected=.02). Finally, brain-behaviour relationships were explored, restricting our anatomical measure of interest to mean qT1 values within bilateral WM. Although no tests passed correction for multiple comparisons, there was a trend association between better delayed recall of Visual Reproduction and decreases in $\mathrm{qT} 1$ of combined $\mathrm{WM}$ on the right hemisphere $(\mathrm{F} 1,11=3.72$, $\mathrm{p}=.08)$, driven by changes in $\mathrm{qT} 1$ of the right fornix $(\mathrm{F} 1,11=4.4, \mathrm{p}=.06)$.

Discussion: This study reveals significant microstructural changes in WM output circuitry of the hippocampus shortly after a FEP. Specifically, increases in $\mathrm{qT} 1$ were found within fimbria, fornix, and mammillary bodies bilaterally. Given that $\mathrm{T} 1$ relaxation times are typically shorter in WM, an increase in qT1 may reflect a combination of decreased myelin content and increased inflammation. Furthermore, preliminary data suggest better visual memory at baseline is associated with lower qT1 within WM microstructure over a 3-month period, suggesting that preserved non-verbal memory ability shortly after a FEP may manifest in a protective anatomical phenotype, particularly within the fornix. Given the importance of the hippocampal-fornix circuit in FEP, both with respect to memory and as a theorized hub of pathophysiology in psychosis, a better understanding of WM microstructure in relation to cognitive profiles in patients may offer a new perspective for treatment targets.

\section{T173. GABA AND GLUTAMATE IN PATIENTS WITH 22Q11.2 DELETION SYNDROME AND HEALTHY VOLUNTEERS AND THE RELATION WITH COGNITION: A RANDOMIZED DOUBLE- BLIND 7TESLA PHARMACOLOGICAL MRS STUDY}

Claudia Vingerhoets ${ }^{*, 1}$, Desmond Tse ${ }^{1}$, Mathilde van Oudenaren², Esther van Duin ${ }^{1}$, Dennis Hernaus ${ }^{1}$, Jan Ramaekers ${ }^{1}$, 
Jaap Janssen ${ }^{1}$, Grainne McAlonan ${ }^{3}$, Oswald Bloemen ${ }^{4}$, Therese van Amelsvoort ${ }^{1}$ ${ }^{1}$ Maastricht University; ${ }^{2}$ Academic Medical Center; ${ }^{3}$ Institute of Psychiatry; ${ }^{4} G G z$ Centraal/Maastricht University

Background: 22q11.2 deletion syndrome (22q11DS) is characterized by a microdeletion on the long arm of chromosome 22. The clinical phenotype of this syndrome is highly variable but symptoms include cognitive impairment, heart malformations, auto-immune problems and a high risk of developing a psychotic disorder. One of the genes located in the deleted region is PRODH which encodes proline dehydrogenase (PRODH). This enzyme is involved in converting proline to glutamate (GLU). GLU is involved in the pathophysiology of psychosis, particularly in cognitive symptoms (Lewis and Moghaddam 2006). Gamma-aminobutyric acid (GABA) is involved in cognition and psychosis as well (Vinkers et al. 2010). With this study we aimed to investigate GLUergic and GABAergic reactivity in the anterior cingulate cortex $(\mathrm{ACC})$ and striatum in medication-free patients with 22q11DS with no psychiatric history and healthy controls (HC).

Methods: This was a randomized double-blind placebo controlled cross-over study. Groups were matched for age and gender. 12 patients with 22q11DS (mean age 35 years) and $20 \mathrm{HCs}$ (mean age 31 years) were enrolled in the study. GABA and GLU, levels in the ACC and striatum were obtained twice with 7Tesla Magnetic Resonance Spectroscopy (MRS, STEAM): once after placebo and once after oral administration of $50 \mathrm{mg}$. riluzole (agent with anti-glutamate and pro-GABA action). Striatal and ACC GLU/GABA ratios were computed as well as GLUergic and GABAergic reactivity (placebo minus riluzole). In addition, within the 22q11DS group, the relationship between cognitive functions (memory and attention) measured with the CANTAB and GABA, GLU, GLU/GABA ratio, GABAergic reactivity and GLUergic in the ACC and striatum were examined.

Results: Analyses of Covariance (ANCOVA) showed no baseline group differences in glutamate and GABA levels and GLU/GABA ratios (corrected for fraction of cerebral spinal fluid, CSF) in both brain regions. A repeated measures ANCOVA showed a trend level significant increase in striatal GABA concentrations after $(p=0.065)$. Riluzole had no significant effect on GLU $(p=0.303)$ and GLU/GABA ratios $(p=0.150)$ in the striatum. No medication $\mathrm{X}$ group interaction effects were found. Riluzole had no significant effect on GABA $(p=0.101)$, GLU $(p=0.847)$ and GLU/GABA ratio $(p=0.108)$ in the ACC. No group main effects and no medication $X$ group interactions effects were found. However, a significant negative correlation was found between verbal memory $(r=-0.650, p=0.030)$ and ACC GLU levels, as well as GLUergic reactivity $(r=-0.733, p=0.010)$. Moreover, in the 22q11DS group, a significant negative correlation was found between attention (target sequence detection $)$ and ACC GLU levels $(r=-0.704, p=0.016)$ as well as GLU/GABA ratio $(\mathrm{r}=-0.602, \mathrm{p}=0.050)$. Furthermore, sustained attention was positively associated with ACC GABA levels $(r=0.700, p=0.024)$ and negatively associated with GLU/GABA ratio $r=-0.639, p=0.047)$ in these patients. Finally, a positive correlation was found between visual memory and striatal GLU levels $(\mathrm{r}=0.616, \mathrm{p}=0.043)$.

Discussion: The present study did not demonstrate differences in ACC and striatal GLU and GABA levels, nor in GLUergig or GABAergic reactivity in response to riluzole between 22q11DS patients and controls. However, these results suggest a role for GLU and GABA in cognition in the 22q11DS group. Therefore, influencing these neurotransmitter systems might enhance cognitive functioning in these patients. More studies are required to replicate these findings.

\section{T174. STRUCTURAL ABNORMALITIES IN THE CINGULATE CORTEX IN ADOLESCENTS AT ULTRA-HIGH RISK WHO LATER DEVELOP PSYCHOSIS}

Adriana Fortea ${ }^{*, 1}$, Phillip van Eindhjoven ${ }^{2}$, Jose Pariente ${ }^{3}$, Anna Calvo ${ }^{3}$, Albert Batalla², Elena de la Serna ${ }^{4}$, Daniel Ilzarbe ${ }^{5}$,
Jordina Tor ${ }^{6}$, Montserrat Dolz ${ }^{7}$, Inmaculada Baeza ${ }^{8}$, Gisela Sugranyes ${ }^{8}$

${ }^{1}$ Hospital Clinic de Barcelona, Universitat de Barcelona; ${ }^{2}$ Radboud University Medical Center, Donders Institute for Brain Cognition and Behavior, Centre for Cognitive Neuroimaging, ${ }^{3}$ Magnetic Resonance Image Core Facility, Institut d'Investigacions Biomèdiques Agustí Pi i Sunyer (IDIBAPS); ${ }^{4}$ Hospital Clinic of Barcelona, Centro de Investigación Biomédica en Red de Salud Mental (CIBERSAM); ${ }^{5}$ Hospital Clinic of Barcelona; ${ }^{6}$ Hospital Sant Joan de Déu, Centro de Investigación Biomédica en Red de Salud Mental (CIBERSAM); ${ }^{7}$ Hospital Sant Joan de Déu, Centro de Investigación Biomédica en Red de Salud Mental (CIBERSAM); ${ }^{8}$ Hospital Clinic of Barcelona, Centro de Investigación Biomédica en Red de Salud Mental (CIBERSAM), Institut d'Investigacions Biomèdiques Agustí Pi i Sunyer (IDIBAPS).

Background: Identification of biomarkers of transition to psychosis in individuals at ultra-high risk (UHR) has the potential to improve future outcomes (McGorry, 2008). Structural MRI studies with UHR samples have revealed steeper rates of cortical thinning in temporal, prefrontal and cingulate cortices in individuals who later develop psychosis in both baseline and longitudinal comparisons (Fusar-Poli, 2011; Cannon, 2014). However, little is known about how onset of prodromal symptoms during adolescence impacts on changes in cortical thickness (CTH) (Ziermans, 2012).

Methods: Multicentre cross-sectional case-control study, including youth aged 10-17 years, recruited from two child and adolescent mental health centres. UHR individuals were identified using the Structured Interview for Prodromal Syndromes criteria with some modifications. Healthy controls (HC) were recruited from the same geographical area. Exclusion criteria comprised personal history of psychotic symptoms, IQ $<70$, autism spectrum disorder, presence of neurological disorder, or antecedents of head trauma with loss of consciousness. The study was approved by the local Ethical Review Boards. All participants underwent a comprehensive socio-demographic and clinical evaluation at baseline and after 6, 12 and 18 months follow-up to identify which individuals converted to psychosis (UHR-P) and which did not (UHR-NP).

High-resolution magnetic resonance structural images were acquired at baseline on a 3Tesla and 1.5Tesla scanners. An inter-site compatibility study was conducted with healthy controls which revealed high inter-site correlation coefficients $(r>.6)$ for CTH measures. Images were pre-processed employing automated procedures implemented in FreeSurfer 5.3.0, cortical parcellation employed the Desikan-Killiany brain atlas. Analyses: First, mean global and lobar (frontal, parietal, temporal, occipital, insula and cingulate) $\mathrm{CTH}$ measurements were computed. Then, within lobes showing group effects, CTH was measured for each parcellation. ANCOVA was performed to test differences between groups in SPSS 22.0, including gender, age, total intracranial volume and site as covariates. Significance was set at $\mathrm{p}<.05$, corrected using the false discovery rate (FDR).

Results: 122 subjects were included (59 UHR-NP vs. 18 UHR-P vs. 45 HC, mean ages: $15.2 \pm 1.5$ vs. $15.0 \pm 1.8$ vs. $15.8 \pm 1.5, \mathrm{~F}=1.9, \mathrm{p}=.15$; gender ( $\%$ female): $61.0 \%$ vs $61.1 \%$ vs $68.9 \%, \chi 2=.76, p=.68$ ). There were no significant differences in case-control proportion between centres: $\chi 2=1.3, \mathrm{p}=.25$. No significant differences in global CTH in UHR-P $(2.57 \pm 0.13 \mathrm{~mm})$ relative to UHR-NP $(2.56 \pm 0.11 \mathrm{~mm})$ and $\mathrm{HC}(2.58 \pm 0.09 \mathrm{~mm})$ were found. There was a significant group effect on the right cingulate cortex $(\mathrm{F}=6.6, \mathrm{pFDR}=.024)$ : UHR-P showed lower $\mathrm{CTH}$ in this area relative to controls $(p=.007$ uncorrected). Within the right cingulate cortex, a significant group effect was found in the posterior cingulate $(\mathrm{F}=5.7$, $\mathrm{pFDR}=.016)$ and isthmus $(\mathrm{F}=4.6, \mathrm{pFDR}=.024)$, and a trend level in the caudal anterior cingulate $(\mathrm{F}=2.9, \mathrm{p}=.057)$ : with smaller $\mathrm{CTH}$ in UHR-P relative to $\mathrm{HC}$ in the isthmus cingulate $(\mathrm{p}=.025)$ and the posterior cingulate $(\mathrm{p}=.066)$. No significant differences were observed between UHR-P and UHR-NP groups. 